not as dramatic as those observed when the Cajal-Retzius cells were ablated, innervation of the SLM and the OML was markedly reduced, and the axons again failed to become branched. Moreover, analysis of the reeler mutant mouse showed a similar defect in formation of the EHP.

It is tempting to conclude that Reelin may act as a signal that specifies the patterning of the EHP, but a degree of caution is warranted. First, the effects of ablating the Cajal-Retzius cells are much more dramatic than the effects of the reeler mutation, suggesting that the consequences of functionally removing these cells cannot be entirely due to the loss of Reelin. Second, the defect in formation of the EHP in the reeler mouse seems to be temporary - although the EHP is less developed on the day of birth, by postnatal day 12 , innervation by the entorhinohippocampal axons is indistinguishable from controls. In fact, the reeler mutant mouse can be interpreted as having a defect in branching of EHP axons within the SLM and the OML, rather than in hippocampal innervation. Nevertheless, there is good evidence that development of the EHP does require Reelin, and that this function is distinct from the part that Reelin plays in cell migration (movement of the cell body).

The involvement of hippocampal Cajal-Retzius cells in formation of the EHP is reminiscent of the way in which subplate neurons form a template for connections in the cerebral cortex ${ }^{6,7}$. Normally, axons from the thalamus relay sensory information to the cortex through the thalamocortical projection. During development, as thalamic axons grow towards their cortical targets, they interact closely with the subplate neurons that line their path. When these neurons are ablated by injecting kainic acid into the developing cortex, the thalamic axons fail to innervate their appropriate cortical targets. So interactions between the thalamic axons and the subplate neurons are required for proper development of the thalamocortical projection. Moreover, the subplate neurons belong to a population of cortical neurons that are generated at an early stage of development and do not participate in the functional circuits of the mature cortex. The similarities between this system and development of the EHP indicate that interactions involving transient neuronal populations may be gener-

\title{
Spot the referee
}

\section{IMAGE \\ UNAVAILABLE FOR COPYRIGHT REASONS}

THIS completely unscientific contest was announced in Nature 384, 214 (1996), and drew on an old practice in British newspapers, the spot-the-ball competition. It had as its immediate inspiration a paper in the British Medical Journal, in which editors of the journal Psychological Medicine described a survey of the ability (or as it turned out, the lack of it) of authors of papers to identify the referees of the paper from their anonymous reports.

The spot-the-referee challenge for Nature readers was to identify the position, in $x, y$ coordinates, in millimetres, of the referee in a picture of a soccer match from which his image had been removed. The specific target was the referee's nose. Here he is shown back in the action, and the official measure of the position of his nose is $x, 40.5 ; y$, 28. Entries came from 18 countries. But all four of those who came closest, with estimates $0.5 \mathrm{~mm}$ out on the $x$ axis and exact on the $y$, are in the United States - James Binley (Aaron Diamond AIDS Research Center, New York), Roberto Fernandez-Larsson (Children's National Medical Center, Washington DC), Scott A. Loiler (University of Massachusetts Medical Center, Worcester, Massachusetts) and Jijun Wan (University of California, San Diego). As in sport, but happily not in science, the referee's decision in this competition is final. Congratulations to the four winners (who will receive a year's subscription to Nature), commiserations to those who came close, and thanks to all who entered. ally required for the patterning of connections in the mammalian brain.

Given that Reelin directs the development of hippocampal connections ${ }^{1}$, might it also be involved in the patterning of thalamocortical connections? This seems to be unlikely as subplate neurons do not express Reelin ${ }^{4,5}$, and thalamic axons can innervate the appropriate cortical targets in the reeler mouse. But this does not rule out the possibility that Reelin helps to regulate some other aspect of cortical connectivity. In the developing cortex, Reelin is expressed by cortical Cajal-Retzius cells known as the marginal-zone cells, which are generated at the same early stage of development as the subplate neurons. The marginal zone subsequently develops into a rich synaptic zone containing the dendrites of cortical pyramidal cells as well as axons from cortical and thalamic neurons. So the patterning of connections within the marginal zone could require the marginal-zone cells from the early stages of development, and it would be interesting to find out whether Reelin is involved in the development of these connections.

So Reelin - a protein that was initially associated with cell migration - now also seems to regulate aspects of axonal growth and guidance. This is particularly interesting in view of studies indicating that cell migration and growth-cone movement probably involve common intracellular mechanisms ${ }^{8}$. The experiments described by Del Río et al. ${ }^{1}$ may be the first evidence that cell migration and axon guidance not only share intracellular molecular mechanisms, but that they might even be regulated by the same extracellular cues. It may not be too farfetched to suggest that recently identified axonal-guidance cues, such as netrins and semaphorins ${ }^{9}$, could also regulate aspects of cell migration. In that case, two of the central aspects of nervous-system development, cell migration and axon guidance, could, in fact, be cellular manifestations of a common set of molecular interactions.

Anirvan Ghosh is in the Department of Neuroscience, The Johns Hopkins University School of Medicine, 725 North Wolfe Street, Baltimore, Maryland 21212, USA.

\section{Corrections}

- IN "The sharp end of quasars" by Patrick $S$ Osmer (Nature 384, 415-416; 1996), in the sentence, "They were able to identify all the radio sources either as galaxies or as stars, and they conclude that none are quasars with $z>5$ ", the word "stars" should read "quasars".

- In "A suspicious signature" by Adriano Aguzzi and Charles Weissman (Nature $\mathbf{3 8 3}$, $666-667 ; 1996)$, the source of the personal communication relating to the 'target-cell hypothesis' was Rudolf Karl Meyer, not K. H. Meyer as stated. 